\section{Cuestiones de género: mujeres en la historia de la música}

Gender issues:

Women in music history

SANDRA SOLER CAMPO sandra.soler@fje.edu

Universidad Rovira Virgili
$\Rightarrow$ Recibido 16/09/2017 Historia

$\checkmark$ Aceptado 30/10/2017

\section{Resumen}

La historia de la música ha mantenido al margen a un gran número de mujeres que se han dedicado a la música. Este prejuicio cultural se ha transmitido de generación en generación y ha dañado gravemente el sexo femenino. El peso de la cultura, tan importante en muchas de nuestras decisiones vitales, ha condicionado a las mujeres en ciertas ocasiones. Así, pocos compositoras están incluidos en los manuales musicales de nuestras instituciones educativas (escuelas, universidades, conservatorios ...). Los docentes tienen un gran reto entre manos ya que pueden ayudar a que esta situación cambie. Los alumnos son protagonistas en este proceso de cambio y pueden cambiar esta realidad androcéntrica en la cual las mujeres son casi inexistentes en determinados campos musicales.

\section{Palabras clave}

Mujeres · Música · Educación · Género 


\section{Introducción}

A lo largo de la historia, las mujeres han contribuido en las artes de modos muy diversos. Notables mujeres artistas a lo largo de los siglos han quedado en un segundo plano e incluso han sido olvidadas. Son muchas las mujeres que han escrito, creado y realizado algunas de las obras más inspiradoras de la historia. Sin embargo, la mayor parte del tiempo, es tas mujeres han sido olvidadas a lo largo del camino.

A lo largo de centenares de años, las mujeres no han tenido a su alcance los medios necesarios ni la formación adecuada para desarrollarse en el mundo musical. Si miramos atrás en el tiempo tanto en el Renacimiento como en el pos terior periodo Clásico, sólo aquellas mujeres que pertenecían a familias nobles o a la aristocracia podían ejercer como músicos. Eso sí, en su entorno doméstico. Hacerlo en la esfera pública era impensable. Ya en el periodo Romántico contamos con un mayor número de mujeres docu mentado como por ejemplo Clara Wieck Schumann (mujer del pianista y compositor Robert Schumann); Alma Mahler (mujer de Gustave Mahler), etc. A pesar de que no hay duda alguna del talento de estas mujeres, siempre quedaron en un segundo plano. A la sombra de sus esposos o hermanos (como fue el caso de Fanny Mendelssohn, hermana de Félix Mendelssohn). Todavía en el siglo XIX para que una mujer pudiese publicar sus propias composiciones tenía que hacerlo bajo un pseudónimo masculino. No estaba bien visto que ellas pudiesen interpretar para un amplio público, ni mucho menos componer y publicar sus creaciones artísticas. Aparecer en la escena pública era cosa de hombres, los cuales no tenían ningún tipo de prejuicio ni inconveniente de mostrar al público qué sabían hacer o cómo hacían "hablar" a los instrumentos (en el caso de los intérpretes).

Durante muchos siglos, ha existido la creencia de que, más allá de la biología las mujeres y los hombres poseen esencialmente diferentes capacidades y funciones. La comprensión de este supuesto ayuda a dar sentido a la perpetuación e in cluso institucionalización de sexo masculino (frente al sexo femenino) en relación con las expectativas de comportamiento, posición dentro de la familia, derechos, la situación pública, la educación, y tipos de trabajo. Esta situación ha dado lugar a la
Women · Music · Education · Gender 
subordinación de la mujer en la sociedad. La musicóloga Lucy Green, en su obra Música, género yeducación (1997), intenta mostrarnos que tanto las prácticas musicales de las mujeres como los significados musicales marcados por el género se han perpetuado a lo largo de la historia en relación al concepto de patriarcado musica y la teoría de la construcción social del significado musical: "Tras la música, hay un hombre; el perfil cerebral de la composición sigue llevando una abrumadora cantidad de connotaciones masculinas" (Green, 1997: 54).

Conocer las circunstancias sociales, políticas y/o económicas de cada periodo histórico, nos ayudará a comprender mejor nuestro pasado, y el del contexto en que mujeres compositoras, intérpretes y directoras desarrollaron su labor artística. Por ello, conocer las diferentes etapas históricas, y el contexto social en el cual se ha desarrollado la actividad musical en todas ellas, hará posible que podamos comprender mejor la situación en la que se encuentran hoy día las mujeres que deciden estudiar música y que posteriormente se insertan en el mundo laboral.

La presencia de las mujeres en el ámbito musical no es reciente. Además, la situación y sociedad en la que se desarrollan, no será la misma en todos los periodos históricos. Si bien es cierto que ha estado en la sombra durante siglos, debemos tener presente que en determinados periodos de la historia ha gozado de mayores reconocimientos. Por ejemplo, durante la civilización egipcia, griega e incluso en algunas cortes renacentistas y barrocas, las mujeres se han podido sentir incluidas en los diferentes contextos musicales. También en las sociedades no industrializadas, las mujeres participaron activamente en la creación musical. Una de las primeras compositoras de la historia de la música de las que tenemos constancia es Safo (Éreso, 600 a. C.), considerada una de las mejores poetisas de la antigüedad. Las poetisas de este periodo (además de leer) cantaban y se acompañaban con instrumentos musicales como la cítara, la lira y/o la flauta. La creadora de estas composiciones líricas se encargaba también de componer la música e incluso los pasos de danza. Pero es a partir del siglo IX d. C (a excepción de algunos casos concretos como la mencionada Safo) en adelante cuando tenemos más información acerca de la participación de la mujer en la música. Dicha información, a pesar de los esfuerzos realizados, continua siendo escasa.
La red de relaciones que constituye la música es muy compleja y significativa. Como seres humanos, somos seres sociales, y toda vida social (independientemente al género al que pertenezcamos) está impregnada de música desde el momento en que nacemos y durante toda nuestra vida. La existencia de varias musicologías ha puesto de relieve la idea de que no hay una sola música importante, sino que cada una de ellas debe valorarse en los términos de su propia cultura. Además, como la música es ambigua y abstracta, puede dar lugar a varias opiniones y a más de un significado diferente. Denominaremos a este hecho pluralismo metodológico o historiográfico de la Musicología actual'.

\section{Musicología feminista}

El discurso feminista y el movimiento social (el cual se identifica con dicho discurso) necesitan una nueva realidad que se va construyendo, la sociedad. La teoría feminista se configura como marco de interpretación de la realidad que nos rodea, la cual visibiliza el género como una estructura de poder ${ }^{2}$. Al conceptualizar la realidad, la teoría feminista pone al descubierto los elementos de subordinación y desventaja social que privan de recursos y derechos la vida de las mujeres.

El feminismo formó parte como disciplina en la musicología en la década de los 70 del pasado siglo. Fue en gran parte gracias a la atención que algunos musicólogos prestaron a la participación de la mujer en la música a lo largo de la historia. James R. Briscoe publicó en 1987 dos antologías de mujeres compositoras titulado Antología de Música de mujeres y Antología Contemporánea de música de mujeres. Karin Pendle produjo y editó una colección de ensayos sobre mujeres en la música Mujeres

1 Las conexiones intradisciplinares también se dan con la historia de la familia, con la historia regional (Ferra de Bartol y García, 1985), la historia de las mentalidades y con el modelo del pluralismo cultural (Sábato, 1988) como el que ha postulado Hilda Sábato, enfoques relativamente recientes de la nueva historia, para adoptar categorías teóricas y métodos a fin de configurar una historia de la música que amplíe las fronteras disciplinarias iniciales y dialogue con la historia de la música nacional.

2 El movimiento feminista, surgió en la década de 1840 en Estados Unidos. En concreto en la primera conferencia feminista en Séneca Falls (Nueva York). El movimiento feminista, buscó aumentar la felicidad y libertad de las mujeres, en un mundo sin opresión. (Riegel, 1965). 
y música: Una historia. Varios musicólogos van redescubriendo la música de mujeres compositoras como Hildegard von Bingen, Barbara Strozzi, Clara Schuman... Gracias a esta labor realizada por musicólogos de todo el mundo, las compañías de música venden en la actualidad más partituras y grabaciones musicales de mujeres compositoras.

Una de las aportaciones más importantes de las activistas feministas ha sido el giro epistemológico que ha hecho que muchas estructuras se hayan tambaleado. La crítica del arte (desde la perspectiva de género) ha introducido la voz y mirada de sujetos que anteriormente han sido excluidos del espacio y del discurso público (Mulvey, 1975).

Según R. Williams, se asegura la hegemonía cuando la cultura dominante utiliza la educación, la filosofía, la religión, la publicidad y el arte para lograr que su predominio les parezca natural a los grupos heterogéneos que constituyen la sociedad, (Miller, T. y Yudice, G., 2004). No obstante, tal renovación epistemológica se diluye en el paradigma del canon ${ }^{3}$. Cuestionar el canon es poner en entredicho el relato aceptado. De este modo, se abre la posibilidad a plantear nuevas narrativas. Nochlin, L. (1971: 2) añade que una crítica feminista de la disciplina es necesaria para poder perforar sus limitaciones culturales e ideológicas, revelar diagonales e insuficiencias no simplemente respecto a la cuestión de las artistas mujeres, sino en la reformulación de cuestiones cruciales para la disciplina en su totalidad.

Debemos tener en cuenta, que a pesar de que la musicología feminista ha trabajado en concordancia con las teorías feministas tradicionales, el nacimiento del feminismo en música ha sido posterior. Por ello, podemos decir que se trata de una ciencia que es relativamente nueva. Se sitúa en el centro del posmodernismo y critica el positivismo desarrollando un nuevo concepto de obra generalizada del patriarcado, el aburguesamiento que refleja la tradicional historia de la música culta y el eurocentrismo. La sociedad patriarcal, se ha encargado de definir qué es lo masculino y qué es lo femenino.

3 El canon es la norma que produce la hegemonía politicosocial y que genera categorías binarias de desigualdad: dentro/fuera; masculino/femenino; alta/baja cultura, etc., estableciendo una estructura de dominación, exclusión y marginación sobre lo que no está aceptado como norma. Destruir esta concepción del canon obliga a modificar la estructura y a construir nuevas perspectivas sobre la definición, análisis e interpretación de lo artístico.
Esta distribución se realiza de un modo desigual, puesto que al sexo femenino lo sitúa en una posición inferior respecto al masculino.

Los estudios feministas se engloban dentro de la corriente postestructuralista denominada Nueva Musicología. Esta co rriente rechaza el análisis realizado hasta el momento, el cual considera la música un elemento aislado el cual se crea y consume. El término Nueva Musicología es acuñado en 1990 por Lawrence Kramer.

La musicología feminista se propone fundamentalmente los siguientes objetivos:

- Restaurar la posición de la mujer a través de la historia compensatoria ${ }^{4}$.

- Investigar si existe un lenguaje musical femenino distinto al masculino.

- Género y organología, formas musicales, tímbrica vocal, significados atribuidos según los parámetros establecidos.

4 Historia Compensatoria: es aquella que ha redescubierto a la mujer en todas las facetas musicales desde la Antigüedad hasta nuestro días, desarrollando su labor como compositoras, intérpretes, mecenas, docentes con una recepción clara.
- Criticar al sistema del patriarcado en el ámbito musical.

- Indagar en las construcciones plurales de género. Ello dará lugar a los estudios de género

La revisión que plantea la musicología feminista, pasa por la deconstrucción del discurso tradicional. Aquel que a través de los siglos ha ido transmitiéndose de generación en generación sin apenas modularse. La revisión de este discurso, hará que se planteen cambios (decons trucción) de toda la base del pensamiento occidental. De modo general podemos afirmar que el posmodernismo es una crítica de los valores de la época moderna. A través del intelecto, el posmodernismo señala una primera desventaja: que la esfera corporal se suprime completamente. Los conciertos públicos en el sentido postmodernista, son criticables porque suprimen cualquier reacción corporal.

5 Deconstrucción: Término acuñado por el filósofo post-estructuralista Jacques Derrida. Es un método de trabajo que se basa en ir desmontando los supuestos ideóógicos que subyacen en el pensami puestos ideológicos que subyacen en el pensamienoccidental, basado en la ficción de una identidad completa y monolítica, sin fisuras ni contradicciones. A través de la desconstrucción se van identificando una a una las ideas sobre las que se sostiene este discurso y se pone en evidencia su carácter construido, no natural (Lechte, 1994: 105-110). 
Fue la etnomusicología quien realizó los primeros estudios sobre las mujeres y la música en las diferentes culturas. A posteriori la musicología se dedicó al estudio de la música (en el caso que estamos describiendo de mujeres) en Occidente. Una de las obras pioneras que trata esta temática es el manual de la musicóloga Sophie Drinker, publicado en 1948. A partir de aquí en adelante, fueron varios los autores curiosos por explorar nuestro pasado y buscar información de nuestras compositoras, intérpretes, de las veladas musicales en los salones...A modo de ejemplo, el manual Women making music: The Western art tradition, 1150-1950, de Jane Bowers y Judith Tick.

Las pioneras Jane Bowers y Judith Tick argumentaron la historia de la música no sólo mediante la adición de músicos excepcionales sino a nivel grupal. Ello les ha permitido discutir los roles de las mujeres en el ámbito doméstico (como artistas y maestras) y considerar su ausencia. El ámbito de la interpretación es quizás el área en el que las mujeres han tenido más presencia desde el siglo XVII hasta la actualidad. Lamentablemente (argumentan las autoras) la musicología ha estado más preocupada por la autoría de los textos que la participación de éstas. (Bowers and Tick, 1987).

Otras autoras como Bloom (1994), consideran que la formación del canon musical sugiere que los estudios de género pueden llevarse más allá de la simple documentación de mujeres en la música (a pesar de su valía). Deben tenerse en consideración también los constructos de género en música y sus instituciones.

Suzanne Cusick (2015), identifica la doctrina de la música por sí misma, como la última de las cuestiones del feminismo. Añade la autora que la imagen de autonomía se asigna a las normas culturales de la masculinidad. Con el fin de descubrir la codificación de género integrada en la idea de música por sí misma, necesitamos examinar las premisas de la teoría musical. (Cusick 2011). Cusick muestra claramente cómo la musicología es una disciplina percibida como masculina (o denominémosla masculinizada). Cuando se fundó la Sociedad de Musicología de Nueva York en 1934, Ruth Crawford fue excluida de la inauguración. Muchos años después, expone Cusick, Charles Seeger confesó en una entrevista que la supresión de la contribución de Crawford fue un intento deliberado de evitar las críticas de que la musicología era también el trabajo en el que las mujeres podían ser partícipes. En resumen, Crawford fue excluida de un evento que fue decisivo en la elaboración de una disciplina que trata de controlar un objeto estetizado cuando se resistía a su atractivo físico.

Una de las conclusiones a las que llega la periodista Amelia Die Goyanes (1998) es que la mayor parte de las ocasiones en que la mujer ocupa un papel musical, lo hace desde una posición que se aleja de las funciones que conllevan poder en cualquiera de sus dimensiones. Tradicionalmente, dentro del plano musical, las posiciones que eran dotadas de un mayor estado de poder eran las de compositor y director; razón por la cual ha resultado de mayor dificultad encontrar sujetos femeninos que ejercieran dichas funciones.

En los textos de canciones o lieder, encontramos a menudo que las mujeres aparecían como culpables de la mala suerte del protagonista. Teniendo en cuenta que nuestra cultura es patriarcal, tiene sentido que nuestra lengua sea sexista. El lenguaje refleja la dicotomía entre lo femenino y lo masculino. El feminismo critica no sólo el lenguaje que refleja el dominio del patriarcado, sino también las estructuras escondidas de segunda fila que afirman esa desigualdad. Por ejemplo, tal y como ejemplifica Gutman (2013) el concierto. La subordinación en el concierto clásico no es exclusivamente de la mujer, sino también del hombre: todos los que forman el auditorio se someten al rito de celebrar un concierto y aceptan o han interiorizado las estructuras que hacen posible tal acto. Sin embargo, es evidente que en la mayoría de los casos los directores de orquesta son hombres que dominan tanto la orquesta como el auditorio.

Dos de las musicólogas más representativas en el campo de la musicología feminista son Susan McClary y Marcia J. Citron. Marcia Citron, se doctoró en California en 1971. Del mismo modo que muchas musicólogas feministas, se introdujo en los estudios de investigación de mujeres y música, y paulatinamente se interesó más por temas que estaban emergiendo como la musicología feminista. Ha contribuido en numerosas publicaciones en este campo. Ha realizado una importante investigación del papel de las mujeres en la música desde mediados de 1970. Citron expone que la música se crea fuera de los contextos sociales específicos. Es decir, una música determinada, puede 
aportarnos una valiosa información acerca de la cultura de la que procede.

La música es capaz de representar un fenómeno social, por ello, podemos afirmar que la música es cultura. Desde el inicio de la humanidad, la música es una de las manifestaciones más importantes dentro del contexto cultural de cualquier época o civilización. Tanto McClary como Citron, han demostrado gracias a sus investigaciones que la historia de las mujeres en la música no ha sido la misma que la historia de los hombres. Los estudiosos continúan investigando la musicología feminista con el objetivo de que la historia de las mujeres y lo que denominamos femenino en la música no sean olvidadas. Citron denomina a la inseguridad que la mujer ha tenido a lo largo de siglos ansiedad de la autoría y la atribuye a la falta de tradición por no formar parte del canon musical. (Citron, 1993: 54).

En la crítica de Susan McClary (2002) vemos un acercamiento desde dentro de $\propto$ la música. La autora señala que incluso la música lleva estructuras del sistema dominante, es decir, del patriarcado. La Teoría Musical del s. XVIII muestra en su lenguaje musical un concepto patriarcal cuando asocian las tonalidades menores a lo fe- menino y las Mayores a lo masculino. La relación de los sexos fue considera desde el s. XVIII como algo natural; la naturaleza femenina y la masculina estaban opuestas de modo dicotómico. Los teóricos del género argumentan contra las reducciones de las formas clásicas (sobre todo la forma sonata) que sean de valor neutral. Basándose en trabajos de narratólogos, Susan McClary (1995) argumenta que el modelo normativo de la forma sonata, sugiere un varón activo frente a una dócil mujer (típicamente un segundo tema de la forma sonata). McClary ha recurrido al examen de los códigos semióticos que en la mayoría de los casos son utilizados en la teoría de la música para describir el segundo tema, y frecuentemente se asocian con la feminidad y fragilidad.

Una de las propuestas por parte de las musicólogas feministas que se ha llevado a cabo ha sido la redefinición de algunos conceptos. Uno de ellos es la categoría varón (como bien social y como bien dominante). Gracias en gran parte a la redefinición de algunos vocablos y conceptos y el hecho de recopilar tantos nombres, obras y datos de mujeres que se dedicaron a la música en los diferentes periodos de la historia, se consiguió revisar el New
Grove Dictionary of Music ${ }^{6}$, y la compilación del New Grove Dictionary of Women Composer. Se trata de un diccionario enciclopédico que recoge a un gran número de músicos (hombres y mujeres). Es junto con el Die Musik in Geschichte und Gegenwart (la mayor enciclopedia alemana de música) una de las mejores fuentes de referencia de la música Occidental.

El objetivo de dicha redefinición de conceptos, era realizar una deconstrucción del discurso musical tradicional y consecuentemente de la base del pensamiento occidental. En este sentido, el investigador Chris Small (1998), considera que la música sólo tiene sentido cuando se interpreta (bien en directo o grabada). Por ello, acuña el término musicking en 1995. Éste término engloba a todas las personas que forman parte del acto musical: desde el personal de limpieza de la sala donde se realiza el concierto, a los intérpretes, al público, a quienes venden las entradas...

6 The Grove Dictionary of Music and Musicians (Dic cionario Grove de la Música y los Músicos) es un diccionario enciclopédico de música y músicos, considerado por muchos estudiosos como la mejor fuente referencial en su tema en inglés. Inicialment realizado con la visión y el estilo de George Grove. Posteriormente adquirio una nueva talla con Stanley Sadie.

\section{Los estudios de género en la} Educación Musical

Los principales ámbitos para situar las investigaciones de los Estudios de Género en la Educación Musical son:

1. La investigación compensatoria.

2. La relectura histórica.

3. La investigación sobre el proceso de enseñanza-aprendizaje.

4. La asignación de roles de género y la construcción de identidades de forma equitativa.

1. La investigación compensatoria tiene como objetivo compensar los vacíos generados desde la tradicional perspectiva androcéntrica. Aquí se incluyen los trabajos que han recuperado nombres de multitud de mujeres que hasta hoy desconocíamos o conocíamos bien poco. En la actualidad, la tendencia es incluir la Pedagogía Feminista dentro de la Pedagogía Tradicional. Uno de los primeros trabajos compensatorios dentro del ámbito educativo-musical fue el realizado por R. Lamb en la década de los 80 . También publicó varias de sus obras más repre- 
sentativas en la década de los 90. Estas publicaciones han hecho posible que un gran número de mujeres compositoras se incluyan en las obras que forman parte de nuestra Historia de la Música. A partir de los años 90 se continuarán realizando publicaciones de esta índole, además de artículos relativos a la Historia de las Mujeres en la Música, los cuales incluyen biografías de compositoras, intérpretes... Información de suma importancia para poder elaborar una práctica docente compensatoria, como por ejemplo el artículo de Roberta Lamb "Feminism as Critique in Philosophy of Music Education" (1994).

2. Uno de los pilares fundamentales en los Estudios de Género es la deconstrucción del discurso androcéntrico. Para ello, será necesario que se pongan en práctica nuevos procedimientos metodológicos con el objetivo de poder realizar nuevas y más 135 completas lecturas. En los últimos años, ha suscitado un gran interés la Historia de la Educación. Gracias a ello, autores como Livingston y Humphreys han hecho posible que se realicen historiografías más equitativas. (Livingstron, 1991). Livingston hace un repaso a aquellas mujeres cuyas vidas puedan servir de modelo a aquellas niñas, chicas y/o mujeres interesadas en carreras musicales. Nombra a Mary Salter, Carrie Jacobs Bond, Eleanor Freer, Amy Beach, Mary Howe entre otras compositoras que han vivido o nacido en el siglo XIX y a Ruth Crawford Seeger, Louise Talma, Marga Richter, Emma Lou Diemer, Nancy Van de vate $y$ otras nacidas en el siglo $X X$ (Humphreys, 1998)

3. Respecto a la investigación sobre el proceso de enseñanza-aprendizaje, debemos tener en cuenta que el docente es una pieza clave en todo proceso de enseñanza-aprendizaje Por ello, es necesario e imprescindible analizar cuáles son sus prácticas. El presente siglo XXI se ha comenzado a trabajar en una línea relacionada con la Pedagogía Emancipatoria. Ésta demanda a los/las docentes una implicaciones individual, es decir que sean críticos/as con los estilos de enseñanza que ellos/as mismos utilizan. Es muy común entre los profesores/as la tendencia de percibir la voz femenina más apta para el canto y facetas interpretativas y relacionar el sexo masculino con facetas creativas y/o otras actividades que relacionan música y tecnología. Es entonces necesario una revisión de éstos (entre otros) planteamientos y expectativas, haciendo que sea posible un acceso más equitativo, libre de prejuicios y que permita superar (o al menos facilitar) la tradicional división de roles. Koza, Morton y O'toole (2005) son autoras que trabajan en este campo. Su conclusión es que los manuales que continuamos utilizando estereotipan la participación de las mujeres en la música. Tras escribir una disertación de música en el siglo XIX titulada Godey's Lady's Book, Koza ha publicado artículos relacionados con la igualdad de género e ilustraciones de los chicos invisibles en los coros escolares. Por su parte Patti O' Toole ha publicado artículos relacionados con las relaciones de poder que se establecen en las agrupaciones corales como Threatening Behaviours: Transgressive Acts in Music
Education (2002), Pholosophy of Music Education Review (2002).

4. Son muchos los trabajos de investigación centrados en la investigación sobre la asignación de roles y la construcción de identidades. Durante varios siglos la práctica de la enseñanza musical ha ido siendo una práctica fundamentalmente femenina. No obstante, los líderes en este sector son (y siguen siendo) los hombres. Las actividades que exigen liderazgo acostumbran a tener mayor presencia masculina. Así, mientras que las mujeres son mayoría dirigiendo corales o enseñando en una escuela, quienes dirigen grandes agrupaciones orquestales son los hombres. Las últimas tendencias de investigación de los Estudios de Género no sólo se centran en las mujeres, sino que incluyen otros colectivos de mujeres e incuso de hombres. En este sentido me gustaría destacar los estudios de hombres ausentes. Entre otras cosas, estos estudios pretenden dar una explicación a la tradicional ausencia del sexo masculino en determinadas facetas musicales, poniendo énfasis a cómo se 
desenvuelven éstos en determinados ámbitos musicales que han sido tradicionalmente femeninos. Los profesores de música deben enfatizar que el canto es una actividad masculina. Los cantantes masculinos adultos necesitan ser introducidos como modelos a seguir. Grabaciones y fotografías de hombres en coros, también pueden ser utilizados para fomentar el interés masculino en el canto. (Kenneth, 1998: 26-32).

\section{Conclusiones}

Durante siglos, se ha perdido (además de ignorado) una valiosa información para poder conocer la historia de las mujeres. Tal y como afirma Virginia Woolf, las mujeres no han tenido el protagonismo creativo que les corresponde (Wolf, 1996). A todas aquellas mujeres que en su momento desafiaron la sociedad en la que se desarrollaron, poniendo de manifiesto su tenemos mucho que agradecerles. Gracias a estas pioneras (a quienes tanto les debemos), las mujeres del siglo XXI podemos desarrollar una labor artística.
Hacer frente a las percepciones y estereotipos que han ido creándose con el tiempo no tiene ni principio ni fin, pero podemos hacer mucho al respecto. No sería del todo cierto atribuir la creación de ciertos estereotipos a las dificultades a las que se han enfrentado las mujeres compositoras, pero no debemos olvidar que tales prejuicios existieron y que debemos continuar luchando para que no sigan existiendo estas diferencias de género (a pesar de lo logrado hasta la actualidad). Lo que sí es cierto es que es necesaria la deconstrucción del entramado ideológico que la sociedad patriarcal ha creado y mantenido a lo largo de centenares de años.

Desde la década de los 70 del pasado siglo XX el arte feminista no ha dejado de expandirse geográficamente. También lo ha hecho a través de la incorporación de nuevas generaciones, tomando nuevas estrategias en sucesivas oleadas llegando a formar una tradición que deja su huella en el arte contemporáneo. No obstante, determinados ámbitos musicales como los campos de la interpretación y dirección orquestal, todavía tienen un largo camino de obstáculos que vencer. Que en pleno s. XXI figuren tan pocas mujeres directoras de orquesta en el ranking mun- dial, (debo destacar a las directoras de orquesta de primera fila Susanna Mälkki y Mirga Grazinyte - Tyla) y el hecho que dos de las filarmónicas más prestigiosas que existen en la actualidad (la Filarmónica de Berlín y la de Viena) no hayan aceptado hasta finales del siglo XX a mujeres en su plantilla instrumental, son hechos que ponen de manifiesto que la integración de las mujeres en este contexto, se está realizando muy lenta y paulatinamente.

La programación 2016/17 del Metropolitan de Nueva York, incluye una ópera compuesta por una mujer. Es la primera que se estrena en este emblemático teatro de ópera neoyorkino después de cien años. Me refiero a L'Amour de Loin de Kaija Saariaho con libreto de Amin Maalouf Que hayan tenido que pasar cien años hasta que un teatro de la embergadura del Metropolitan no estrene una obra compuesta por una mujer, es un hecho que demuestra cuál es la situación de la mujer compositora en la actualidad. La programación de los teatros de ópera teatro, auditorios, continúa incluyendo obras pertenecientes a autores de siglos anteriores que se ofrecen a una audiencia que está acostumbrada a dichas piezas.

Los libros de texto, material que utili- zan la mayor parte de profesorado el día a día en las aulas de nuestro colegios, institutos, escuelas de música, universidades, conservatorios..., continua siendo una herramienta. Es imprescindible que el manual de texto que se use se revise y actualice. Sin embargo, los manuales que se continúan utilizando están sin actualizar y continúan perpetuando la situación androcéntrica. Los libros de historia de la música que todavía se utilizan hoy en día o bien no citan a ninguna mujer o bien lo hacen mediante pequeñas contribuciones (breves biografías generalmente) Dicha contribución es muy breve y pobre. Además, crea en el alumnado (o a cualquier persona que se lea el manual) una sensación de que aquella mujer que se nombra es una excepción

Existen y han existido un gran número de mujeres compositoras, directoras, pedagogas, intérpretes...que deben ser conocidas, explicadas, y que los alumnos conozcan cuál ha sido su aportación en la historia de la música. Del mismo modo que las composiciones de Mozart son explicadas y comentadas en los conservatorios, ¿Por qué no trabajar con la misma profundidad y admiración las obras de Clara Schumann? 
Es complicado identificar cuáles son los prejuicios y estereotipos de género en la educación musical. Por ello:

Es necesario investigar conjunta mente en el currículum oculto de la educación musical que se ofrece en colegios, escuelas de música, conservatorios...para poder activar acciones coherentes y que el alumnado sea consecuentemente capaz de ser crítico con las construcciones de género que él/ella mismo hace del entorno que le rodean y que se sienta partícipe en el proceso de enseñanza - aprendizaje, viendo que sus necesidades, opiniones y valoraciones se tienen en cuenta. Es necesario conocer las controversias, discursos, ideologías y significados musicales existentes para así poder in vestigar sobre sus posibles consecuencias (Castro, 2007: 231-243).

Es casi imposible que en la música contemporánea (la cual engloba muchas y muy diferentes estéticas) no encontre$\propto$ mos nada que no nos interese. Creo que es muy importante dar a conocer aquellas obras musicales compuestas por jóvenes compositoras/es, o compositores que es tán en activo, o bien obras que aún están sin descubrir porque no han sido nunca tocadas o, si se han interpretado, ha sido en contadas ocasiones.

No podemos entender el ámbito de la creación artística sin la labor desempeñada por mujeres. A pesar de que a lo largo de la historia muchas de ellas han sido silenciadas e incluso olvidadas, el camino a seguir (además de recuperar nuestro pasado histórico y premiar la trayectoria de todas las mujeres artistas olvidadas) es la de hacer efectivo el principio de igualdad entre sexos y por lo tanto de oportunidades y abordar este problema social y cultural para no repetir la misma situación (tan desfavorecedora para el sexo femenino) que históricamente se ha ido repitiendo.

No podemos afirmar que existe en el siglo XXI una plena igualdad de sexos en la actualidad en el ámbito musical, puesto que a pesar de los grandes esfuerzos, logros y avances logrados, todavía nos falta un largo camino por recorrer. Como expone Valdebenito, "para generar cambios desde la educación musical en perspectiva de género, estos deberían construirse equilibrando los aportes de las diferentes identidades; deconstruyendo, redefiniendo y tensionando ciertas construcciones estereotipadas de género y generando conciencia crítica de los problemas de poder y de invisibilización perpetuados a lo largo del tiempo" (Bolaño, 2015: 305).

\section{Bibliografía}

Bloom, H. (2015). Poemas y poetas: el canon de la poesía. Madrid: Páginas de es puma.

Bolaño, M. (2015). Los sesgos de género a través del currículum oculto de educación musical. RES, Revista de Educación Social, 21, 300- 314. Miscelánea. Universidad de Santiago de Compostela.

Bowers J. (1987). Women making music: The Western Art Tradition, 1150- 1950. Illinois: Paperback.

Castro, P. (2007). John A. Sloboda. En Díaz M. y Giráldez A. (coords.), Aportaciones teóricas y metodológicas a la educación musical. Barcelona: Editorial Grao.

Cusick, S. (2015). Francesca Caccini at the Medici Court: Music and the Circulation of Power. Chicago: University of Chicago Press.
Die Goyanes, A. (1998). Mujeres en la musica. en Manchado Torres, M., (ed.): Musica y Mujeres. Genero y poder. Madrid: Horas y horas.

Green L. (2001). Música, género y educación. Madrid: Ediciones Morata S.L.

Gutman, L. (2013). Amor O Dominación. vo Extremo

McClary, S. (2002). Feminine Endings: University of Minnesota Press.

Small, C. (1998). Musicking: The Meanings of Performing and Listening. London: Paperblack. Los estragos del patriarcado. Madrid: NueMusic, Gender and Sexuality. Minessota: 